\title{
Y a-t-il une logique de l'absence dans la ponctuation d'Yvain du manuscrit BnF Fr. 12560 ? Note méthodologique
}

\author{
Nicolas MAZZIOTTA \\ Université de Liège \\ nicolas.mazziotta@uliege.be
}

\section{Resumen}

Este artículo pretende demostrar que la consideración de usos puntuarios interesantes pero aislados en los textos medievales lleva en ocasiones a conclusiones precipitadas. Analizamos, en concreto, la puntuación de Yvain en el manuscrito BnF Fr. 12560, códice en el que los versos se hallan puntuados de modo prácticamente sistemático a final de verso. Los llamativos casos en que se produce ausencia de puntuación en ese contexto parecen responder a una primera explicación: tal ausencia se debería a la presencia de palabras gramaticales y a una forma de continuidad sintáctica. Pero esta explicación no es suficiente, cuando contrastamos los casos de ausencia y los casos de presencia de puntuación, en un estudio del texto en su conjunto.

Palabras clave: puntuación, francés antiguo, estadística, Yvain, Chrétien de Troyes.

\section{Résumé}

Dans cette contribution, il est montré que la prise en considération exclusive de détails intéressants mène à des conclusions hâtives. Quand on examine le texte d'Yvain dans le manuscrit BnF Fr. 12560, on observe que ce dernier est ponctué de façon pratiquement systématique en fin de vers. Les cas où la ponctuation manque attirent l'œil et réclament une explication. En observant uniquement ces cas, on a l'impression que cette explication est claire : c'est la présence de mots-outils et celle d'une forme de continuité syntaxique qui expliquerait cette absence. Toutefois, quand on prend en considération l'ensemble du texte et que l'on met en contraste les cas où la ponctuation manque et ceux où elle est présente, il n'est plus possible de tirer de conclusions.

Mots-clés : ponctuation, ancien français, statistiques, Yvain, Chrétien de Troyes.

\section{Abstract}

This paper investigates the punctuation of Yvain in the manuscript BnF Fr. 12560 and demonstrates that focusing on interesting details only leads to misleading conclusions. Most

\footnotetext{
*Artículo recibido el 15/09/2020, aceptado el 30/03/2021.
} 
verses are marked by a final punctuation mark. The more seldom cases that display no punctuation mark draw attention and require an explanation. By examining only these specific cases, we could think that the presence of function words and the continuity of syntactic structures from one verse to the next are valid explanations. However, such conclusions do no hold if the whole text is taken into account. Indeed, the contrast between the presence and the absence of punctuation is not strong enough.

Keywords: punctuation, Old French language, statistics, Yvain, Chrétien de Troyes.

\section{Introduction}

La règle dite « de Betteridge » dans le milieu journalistique pose que si un titre se termine par un point d'interrogation, la réponse à la question posée est probablement " non ». Cette contribution présentera la raison pour laquelle, à la question «Y a-t-il une logique de l'absence dans la ponctuation d'Yvain du manuscrit BnF Fr. 12560 ?", nous estimons préférable de répondre: "Je ne sais pas " ${ }^{1}$. Notre objectif est ici principalement méthodologique. Il s'agira de montrer que le recours à des statistiques élémentaires nous protège de la tentation d'adhérer hâtivement à des explications séduisantes intellectuellement. Il nous paraît d'autant plus important d'insister sur la méthodologie que les travaux récents sur la ponctuation sont loin d'être infaillibles de ce point de vue et d'un point de vue théorique - voir la critique de Cinato (2019) sur les études publiées dans Fasseur et Rochelois (2016).

Cette contribution porte sur l'absence de ponctuation en fin de vers dans la version d'Yvain (Le chevalier au lion) de Chrétien de Troyes, telle que nous la livre le manuscrit BnF Fr. 12560 (manuscrit champenois datant du milieu du XIII ${ }^{e}$ s.). La transcription de ce manuscrit est mise à disposition dans Kunstmann (2001), qui néglige volontairement la ponctuation. Les exemples cités ci-dessous sont tirés de cette édition, à laquelle nous avons ajouté la ponctuation originale que nous avons observée sur le manuscrit. La version de ce manuscrit constitue, aux yeux de la critique philologique, une assez bonne copie malgré quelques passages obscurs (Woledge, 1986).

Dans la section 2, nous présentons le cadre théorique, les résultats antérieurs et les principaux faits connus à propos de la ponctuation du français médiéval et de la ponctuation de ce manuscrit en particulier. Nous définissons également la question que pose cette absence de ponctuation. La section 3 présente les premiers résultats et ébauche une explication apparemment cohérente et séduisante. La section 4 met en doute ces premiers résultats et montre, à l'aide d'outils statistiques simples, que l'explication avancée dans la section précédente n'est pas satisfaisante. La conclusion synthétise la démarche et son apport méthodologique.

\footnotetext{
${ }^{1}$ Nous remercions Franck Cinato et Julie Glikman pour leur relecture et leurs suggestions.
} 


\section{Définition de la question}

Un certain nombre d'études sur la ponctuation médiévale ont montré la manière dont les signes de ponctuation s'articulent aux unités qui constituent leur contexte linguistique, dans une perspective proche de celle de Malcolm B. Parkes (1992), qui considère les signes de ponctuation comme des outils de désambiguïsation - on remarque d'ailleurs régulièrement que leur absence peut poser des problèmes de lecture (cf., par exemple, Morrison, 2016). L'article pionnier de Christiane Marchello-Nizia (1978) aborde leur interaction avec des "unités de lecture ": il s'agit d'évaluer le fonctionnement de la ponctuation comme une aide au décryptage du texte. Les thèses d'Alexei Lavrentiev (2009) et de Nicolas Mazziotta (2009) visent à déterminer les paramètres linguistiques qui sont compatibles avec l'utilisation de signes linguistiques : le premier parle d'" unités (linguistiques) ponctuables ", le second de "spécifications facultatives (de constructions syntaxiques)». Nos travaux plus récents (Mazziotta, 2018) sur la ponctuation des énoncés s'achevant en cours d'octosyllabe mettent en évidence que l'articulation des énoncés est favorable à l'apparition de signes de ponctuation (et constitue un " contexte ponctuable»). Cette dernière étude porte entre autres sur le manuscrit que nous étudions ici. Il y est montré que : (i) le système employé par le scribe est d'un très haut degré de régularité, ce qui le distingue des autres manuscrits de la tradition; (ii) la distribution du tracé des signes correspond à des différences de valeur souvent prévisibles. Le scribe de cette copie témoigne d'une conscience métalinguistique développée.

Ces études portent, pour l'essentiel, sur la présence de signes de ponctuation dans un environnement linguistique particulier (souvent dans une optique onomasiologique). Par contre, la question de l'absence de signe n'est que peu soulevée - voir toutefois Mazziotta (2009). La ponctuation demeure une spécification facultative, qui permet d'aider la lecture, mais dont de nombreux scribes ne manquent pas de se passer. Rechercher les "règles" qui gouvernent les absences permet de compléter cette approche. Que dire en effet des cas où c'est l'absence qui revêt un caractère exceptionnel ?

Le manuscrit BnF Fr. 12560 est abondamment ponctué. La plupart des points que le manuscrit contient sont ajoutés en fin de vers de façon très fréquente, comme cela est courant dans la pratique de l'époque (Llamas-Pombo, 2007). Voir Figure 1 et sa transcription partielle (1), par exemple, en cours de ligne 4 et fin des lignes 8-20. 


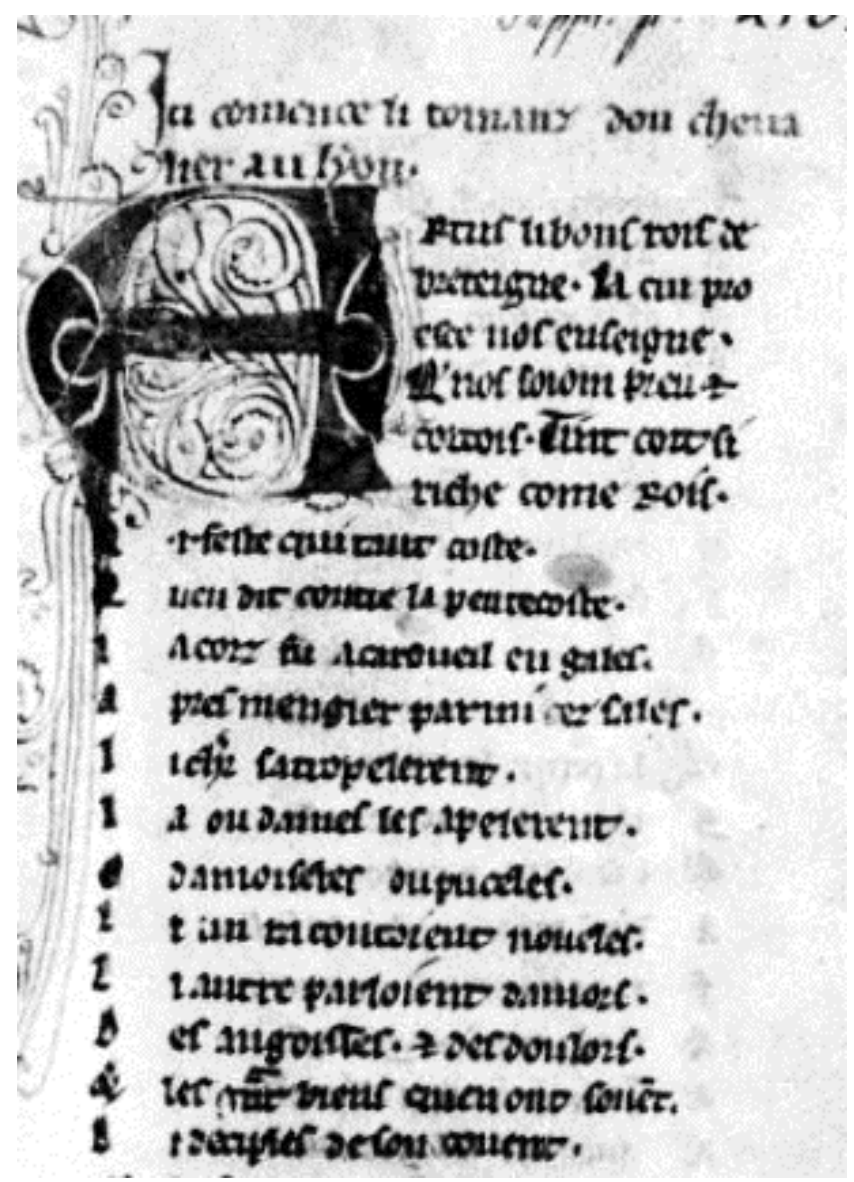

Figure 1. Détail, ms. BnF Fr. 12560, fol. 1b. Ponctuation de la fin du vers.

(C) Bibliothèque nationale de France

(1) Ici comence li romanz dou cheva

lier au lyon.

Artus li bons rois de

Breteigne $\cdot$ La cui pro

esce nos enseigne .

Que nos soiom preu et .

cortois . Tint cort si

riche come rois .

A $\cdot \mathrm{i} \cdot$ feste qui tant coste .

Qu'en dit contre la Pentecoste ${ }^{2}$

La corz fu a Cardueil en Gales . [...]

'Ici commence le roman du Chevalier au lion. Arthur, dont l'excellence nous apprend à être vertueux et courtois, tint une cour d'un luxe digne d'un roi qu'on doit nommer la Pentecôte. La cour se tint à Carduel, au pays de Galles [...]'.

\footnotetext{
${ }^{2}$ Vers probablement fautif. Voir Pierreville (2016 : 112-113) pour cette traduction.
} 
Comme on peut l'observer dans le passage en prose figurant sur la même page et écrit dans la même écriture, identifié comme un fragment de pénitentiel dans la notice de la $\mathrm{BnF}$, le marquage de la fin de la ligne correspond toujours à un autre marquage (chiffres ou articulation entre l'énoncé de la faute et celui de la pénitence). Voir Figure 2 et transcription partielle dans (2).



Figure 2. Détail, ms. BnF Fr. 12560, fol. 1a. Ponctuation de la prose.

(C) Bibliothèque nationale de France

(2) [...] sodomites ou il ait geu a beste char

nelment $\cdot$ il en a $\cdot$ vii $\cdot$ anz $\cdot$ Se nus

fet pollution $\cdot \mathrm{i} \cdot$ foiz par lui me

ifaciet .) il en a $\cdot$ iiii $\cdot$ quaresmes $[\ldots]$

'[...] sodomites ou ait couché avec un animal, il en a pour sept ans. Si quelqu'un se masturbe une fois, et se comporte mal par lui-même, il en a pour quatre carêmes $[\ldots]$.

Le nombre de vers ponctués de la sorte dans Yvain (5920, soit 88\%) est nettement supérieur au nombre de vers non ponctués (821, soit $12 \%$ ). Cela soulève la question de la logique de l'absence d'un signe là où, selon la logique apparemment suivie par le scribe, il «devrait " y en avoir un. En effet, comme on le voit aux lignes 3 et 8 de la Figure 3, l'absence de marquage « saute aux yeux » du lecteur habitué. 




Figure 3. Détail, ms. BnF Fr. 12560, fol. 22d. Absence signe en fin de vers (lignes 3 et 8). (C) Bibliothèque nationale de France

(3) [Le lion resta éveillé] Del cheval garder qui pessoit .

L'erbe - qui petit l'encressoit .

$\mathrm{Au}$ matin s'en revont ensemble

Et itel vie ce me semble .

Come il orent la nuit eüe .

Ont andui emsemble tenue .

Puis trestoute icele semaine.

Tant qu'il vindrent a la fonteine

Et desouz le pin les mena.

'Le lion resta éveillé pour garder le cheval qui broutait l'herbe qui le nourrissait peu. Au matin, ils s'en allèrent ensemble et menèrent, il me semble, la même vie que celle qu'ils avaient eue la nuit, et ensuite pendant toute la semaine, si bien qu'ils arrivèrent près d'une fontaine et que cela les mena sous un pin'.

La question qui se pose est donc ce qu'indique cette absence de marque dans un contexte habituellement ponctué, c'est-à-dire l'unité ou le contexte qui la justifie ou l'impose. Nous allons à présent exposer comment nous sommes passé de l'enthousiasme au doute dans notre démarche visant à déterminer les causes linguistiques de cette pratique.

\section{Enthousiasme}

Dans cette section, nous rendons compte des premières observations que nous avons effectuées au départ d'une intuition : l'absence de ponctuation en fin de vers a l'air associée à la présence d’un mot-outil au début du vers suivant (\$3.1) et à l'indication d'une forme de continuité syntaxique et textuelle qui interagit avec ces 
mots-outils $(\$ 3.2)$. Cela nous mène à la première conclusion, intellectuellement séduisante, selon laquelle la logique de la ponctuation de fin de vers serait l'inverse de celle de la ponctuation interne au vers $(\$ 3.3)$.

\subsection{Présence des mots-outils}

Depuis les premières études sur la ponctuation, il a été remarqué que les motsoutils attirent la présence d'un signe devant eux. Cela a été souligné pour et en particulier (Buridant, 1980), et il s'agit d'une des premières tendances qui a été observée pour différents types de textes (Roques, 1952; Marchello-Nizia, 1978, Boutier, 2001) et qui a été confirmée par les thèses sur la question (Lavrentiev, 2009; Mazziotta, 2009). Or, l'observation d'extraits comme (4) est particulièrement éloquente :

$$
\begin{aligned}
& \text { Si se pooient solacier } \\
& \text { De l'acoler · et del besier } \\
& \text { Et de parler · et de veoir } \\
& \text { Et de delés eles seoir } \\
& \text { Qu'itant en orent il au meins } \\
& \text { (Ms. BnF Fr. 12560, fol.16a) }
\end{aligned}
$$

'Ils peuvent se plaire à les prendre contre eux, les embrasser, leur parler et les admirer et s'asseoir près d'elles; c'est ce qu'ils eurent au moins alors'.

Il semblerait, à première vue, que la logique de la ponctuation de et est inversée suivant que ce dernier figure en début de vers ou en fin de vers. On voit que le et du vers 2 est précédé d'un point et que le vers 3 contient également un point devant le et à l'hémistiche. Par contre, les vers qui précèdent un et initial ne finissent pas par un point.

Le Tableau 1 montre la fréquence cumulée des cas où la ponctuation est absente (sur 821) en fin de vers quand le vers suivant débute par un des mots-outils les plus fréquents; la deuxième colonne présente la fréquence absolue et la troisième la fréquence relative, pour chaque mot-outil :

\begin{tabular}{|c|c|c|}
\hline mot-outil & fréquence absolue & fréquence relative \\
\hline$e t$ & 103 & 0.13 \\
\hline$q u e$ & 75 & 0.09 \\
\hline$s i$ & 36 & 0.04 \\
\hline$m e s$ & 32 & 0.04 \\
\hline$q u i$ & 31 & 0.04 \\
\hline$n e$ & 30 & 0.04 \\
\hline$q u$ & 27 & 0.03 \\
\hline$d e$ & 20 & 0.02 \\
\hline
\end{tabular}




\begin{tabular}{|c|c|c|}
\hline mot-outil & fréquence absolue & fréquence relative \\
\hline$s e$ & 19 & 0.02 \\
\hline$l a$ & 17 & 0.02 \\
\hline TOTAL & $\mathbf{3 9 0}$ & $\mathbf{0 . 4 8}$ \\
\hline
\end{tabular}

Tableau 1. Fréquences de non-marquage avant un vers débutant par un mot-outil.

Près de la moitié des absences correspondent ainsi à une occurrence d'un motoutil qui explique à lui seul $2 \%$ de ce type de cas ou plus. En revenant au texte, on observe que ces mots sont le plus souvent des marques de continuité syntaxique ou d'articulation textuelle. On atteint 401 cas expliqués si l'on ajoute les cas isolés suivants aux mots repris dans le Tableau (les variantes sont indiquées à l'aide de la notation classique des expressions régulières) : qui, cui, don [ct], [ae]in[sz], (c|qu)ar, quand, s'.

\subsection{Continuité syntaxique}

Si l'on considère que l'emploi de ces mots-outils constitue un indice de présence d'une unité ou d'un contexte ponctuable, 420 cas restent inexpliqués. Or, si les motsoutils de la liste ci-dessus sont souvent des indicateurs de continuité et qu'ils correspondent à l'absence de marquage, la suite logique de la recherche consiste à observer les cas non marqués et à vérifier s'ils manifestent des enjambements, des structures continues qui se prolongent sur plusieurs vers.

(5) Et voient dou cheval trenchié

Devant la porte la moitié .

(Ms. BnF Fr. 12560, fol. 8a)

'Et ils voient la moitié du cheval tranché devant la porte'

(6) Qu'encor vaut meuz ce m'est avis

Uns cortois morz c'uns mauvés vis .

Por ce me plest a raconter

Chose qui face a escouter

Del roi qui fu de tel tesmoig .

Qu'en en parole et pres et loing

(Ms. BnF Fr. 12560, fol. 1b)

'Car il vaut mieux, c'est mon avis, un gentilhomme mort qu'un méchant en vie.

Pour cela, je me plais à raconter une histoire qui soit à entendre à propos d'un roi qui fut témoin de choses dont on parle ici et là-bas'.

Ainsi, la structure syntaxique qui court sur plusieurs vers dans (5) est la moitié $d u$ cheval. La majorité des cas résiduels (305 / 420, soit $73 \%$ ) sont effectivement similaires à ceux observés dans (5) et (6), avec des compléments régis par un verbe (raconter chose, escouter del roi) ou un nom ou des circonstanciels dépendant d'un mot figurant au vers précédent. Il s'agit là d'une continuité proprement syntaxique, et non textuelle. 
Un rapide test statistique (à l'aide de la méthode classique du "chi-deux "; Figure 4), effectué sur la répartition des vers non ponctués par rapport à la distribution relative des mots-outils et de la continuité syntaxique, montre par ailleurs une association très claire entre ces mots-outils et la rupture syntaxique (indiquée par la non-correspondance flagrante entre les cadrans du disque). Cela nous laisse penser que ces deux raisons de l'absence de signe sont en quelque sorte complémentaires.

Fins de vers non ponctuées



Figure 4. Représentation de la relation entre la présence de mots-outils et la continuité syntaxique.

Sans entrer dans les détails techniques, le fait que les traits de contour des portions du disque ne se chevauchent pas d'un quartier à l'autre indique une différence statistiquement significative (chi-deux : 36,8 ; probabilité : $1,3 \mathrm{e}^{-9}$ ).

Un retour au corpus nous permet d'observer que les 193 cas de rupture syntaxique associées à des mots-outils correspondent effectivement à des articulations entre énoncés à l'aide des formes et, ne, si, etc. (Voir, par exemple (7), ligne 3).

(7) En touz tens sa foeille li dure .

Qu'il ne la pert por nul iver Et s'i pent $\cdot \mathrm{i} \cdot$ bacins de fer A une si longue chaenne . (Ms. BnF Fr. 12560, fol. 3c)

'Son feuillage dure éternellement, si bien qu'il ne le perd en aucun hiver. Et un bassin de fer y pend, accroché à une longue chaîne.'

\subsection{Première interprétation}

Les premières observations mettent en évidence une claire tendance qui associe l'absence de ponctuation en fin de vers à la présence de mots-outils et à la continuité syntaxique et textuelle. On peut combiner cela aux conclusions antérieures pour tirer les conclusions hypothétiques suivantes : 
(i) En cours de vers, la ponctuation est une marque qui obéit à des règles similaires à celles observées dans la prose : marquage des énoncés (Mazziotta, 2018), lien fort avec les mots outils et en particulier le "strument $e t$ " (Buridant, 1980 ainsi que les autres travaux cités sous $₫ 2$.

(ii) En fin de vers, c'est l'absence de ponctuation qui constitue une marque contrastant avec l'usage régulier du marquage. Ce sont les mêmes unités/contextes que ceux qui attirent la ponctuation en cours de vers qui, au contraire, la rejettent en fin de vers.

\section{Doute}

Pour satisfaisantes que soient les premières conclusions auxquelles nous avons abouti, il nous faut nous poser la question de ce que nous avons réellement observé. En l'occurrence, nous avons évalué la présence et l'absence de mots-outils ainsi que le contraste entre la continuité et la rupture syntaxique et textuelle dans les cas où la ponctuation n'était pas présente en fin de vers. Pour compléter notre étude, nous avons également brièvement examiné le rapport entre la présence de mots-outils et la continuité syntaxique, toujours en cas d'absence de ponctuation en fin de vers.

Dans tous les cas, seule l'absence de ponctuation a été considérée, ce qui implique un manque flagrant de tertium comparationis. Pour que nos observations puissent être évaluées rigoureusement, nous avons besoin d'observer également les fréquences des mots-outils et de la continuité quand un signe de ponctuation est présent en fin de vers. Pour expliquer l'absence de signe, il nous faut déterminer (mesurer) comment elle contraste avec la présence. Il nous faut donc revenir au texte et compter tous les cas, pour tous les vers.

Dans un premier temps, nous pouvons nous concentrer sur les mots-outils. Nos comptages nous mènent à constituer le Tableau 2, qui montre la distribution croisée de ces derniers et des signes de ponctuation.

\begin{tabular}{|c|c|c|}
\cline { 2 - 3 } \multicolumn{1}{c|}{} & Non ponctué & Ponctué \\
\hline Mot-outil & 401 & 2815 \\
\hline Autres & 420 & 3105 \\
\hline
\end{tabular}

Tableau 2. Tableau croisé entre présence/absence de mots-outils et la présence/absence de ponctuation à la fin du vers précédent.

Intuitivement, nous pouvons constater que les chiffres ne manifestent pas d'écarts absolus particulièrement marqués. On s'attend à ce que les tests statistiques ne renvoient pas de résultats significatifs, ce qui est effectivement le cas : le chi-deux associé à ce tableau est très faible $(0,22)$ et il est associé à une probabilité élevée d'acceptation de l'hypothèse nulle $(0,64)$. En conséquence, ce relevé ne prouve absolument pas qu'il y a une relation entre la présence de mots-outils et l'absence de ponctuation au vers précédent - il aurait tendance à démontrer que la relation est aléatoire (ce qui, en toute rigueur, ne peut être véritablement démontré). 
Nous pouvons également réévaluer la continuité syntaxique d'un vers à l'autre. La simple observation des deux premières colonnes complètes du manuscrit révèle 10 ruptures pour 41 vers (folio 1 b) et 14 ruptures pour 38 vers (folio 1c). Sans entrer dans les détails d'un relevé exhaustif (extrêmement coûteux en raison de l'inexistence d'outils informatiques adaptés pour nous aider dans la tâche), il est probable qu'il s'agit d'un phénomène omniprésent chez un auteur par ailleurs adepte de la brisure du couplet, en particulier pour le texte dont nous étudions le manuscrit. En effet, 38,2 \% des couplets sont brisés si l'on se reporte à l'édition de Pierreville 2016 (James-Raoul 2017). Ainsi :

(i) La probabilité que les mots-outils suivent plus particulièrement les absences est ridiculement faible.

(ii) Il est pratiquement certain que la continuité, omniprésente dans le texte à un degré nettement supérieur à celui de l'absence de marquage, n'est pas non plus spécifique à l'absence de marque.

En conséquence, il faut bien admettre que les conclusions précédentes sont bancales, car on y aboutit en considérant comme exceptionnelles des particularités générales du texte (mots-outils en début de vers et continuité d'un vers à l'autre). L'effort fourni pour rassembler les données d'un dépouillement exhaustif concernant la corrélation entre la présence de mots-outils et l'absence de ponctuation n'a servi qu'à invalider nos premières observations, malheureusement trop intuitives.

\section{Conclusion}

Après avoir nourri des espoirs d'explications judicieuses permettant de comprendre pourquoi la ponctuation était absente en fin de vers $(\$ 3)$, nous avons dû nous résigner à admettre que nous n'avons pas abouti à une explication fiable. La prise en compte de l'ensemble des données (et non uniquement des occurrences les plus saillantes) ne permet pas d'obtenir une distribution statistiquement significative (\$4). Nous ne pouvons pas encore répondre à la question " Y a-t-il une logique de l'absence dans la ponctuation d'Yvain du manuscrit BnF Fr. 12560 ? " La question de l'oubli et de l'erreur reste ainsi entière, car s'il est difficile d'ajouter un point incongru, il est aisé d'en omettre par inadvertance.

Il reste cependant que nous pouvons continuer nos recherches avec les acquis suivants, qui n'ont pas été remis en cause :

(i) Les absences de signes sont des saillances qui attirent l'attention dans les textes en vers fortement ponctués en fin de vers.

(ii) Les logiques ponctuationnelles de la prose et du vers different.

Toutefois, décrire les règles qui expliquent l'absence de signes reste un besoin capital pour avancer dans la recherche sur la ponctuation médiévale. Si cette dernière est bel et bien une marque spécifiant une unité ou un contexte ponctuable, nous 
devons, malgré les difficultés, nous efforcer de mettre au jour les unités et les contextes qui, au contraire, y sont rétifs.

Nous tenions à rendre compte de ces résultats mitigés. Pour nous garder de glisser dans le biais cognitif de l'illusion des séries, qui consiste à percevoir des régularités dans des données aléatoires, il nous faut prendre des précautions salvatrices. La focalisation sur les détails néglige l'évaluation de leur spécificité au regard de l'ensemble des données.

Voilà pourquoi, pour pouvoir caractériser un phénomène (comme l'absence de ponctuation) et l'expliquer, il est souvent important de prendre en considération son antagoniste dans toute sa mesure.

\section{RÉFÉRENCES BIBLIOGRAPHIQUES}

BOUTIER, Marie-Guy (2001) : "Études sur des chartes luxembourgeoises », in Kurt Gärtner, Günter Holtus \& Andrea Rapp (éds), Skripta, Schreiblandschaften und Standardisierungstendenzen Urkundensprachen im Grenzbereich von Germania und Romania im 13. und 14. Jahrhundert, Trier, Kliomedia, 419-447.

BURIDANT, Claude (1980) : « Le strument et et ses rapports avec la ponctuation dans quelques textes médiévaux ", in Anne-Marie Dessaux-Berthonneau (éd.), Théories linguistiques et traditions grammaticales. Lille, Presses universitaires de Lille, 13-53.

Cinato, Franck (2019) : "À propos du volume Ponctuer l'auvre médiévale. Des signes au sens (Valérie Fasseur et Cécile Rochelois [éds], Genève, Droz, 2016)». Histoire Épistémologie Langage, 41, 179-191.

FASSEUR, Valérie \& Cécile ROCHELOIS [éds] (2016) : Ponctuer l'œuvre médiévale. Des signes au sens. Genève, Droz.

JAMES-RAOUL, Danièle (2017) : « La brisure du couplet dans Le Chevalier au lion de Chrétien de Troyes». Revue des littératures et des arts, 17. URL: https://revues.univpau.fr/opcit/243

Kunstmann, Pierre (2001): Chevalier au lion. Ottawa, LFA. URL: https://www.francaisancien.net/activites/textes/chevalier-au-lion/index.html

LAVRENTIEV, Alexei (2009): Tendances de la ponctuation dans les manuscrits et incunables français en prose, du XIII au XVe siècle. Volume 1. Thèse de doctorat sous la direction de Christiane Marcello-Nizia. Lyon, École normale supérieure, Lettres et Sciences Humaines. URL : https://halshs.archives-ouvertes.fr/halshs-00846762

LLAMAS-POMBO, Elena (2007) : «Réflexions méthodologiques pour l'étude de la ponctuation médiévale ", in Alexei Lavrentiev (éd.), Systèmes graphiques de manuscrits médiévaux et incunables français: ponctuation, segmentation, graphies. Actes de la journée d'étude de Lyon, ENS LSH, 6 juin 2005. Chambéry: Presses de l'Université de Savoie, 11-48.

MARCHELlO-NizIA, Christiane (1978) : "Ponctuation et "unités de lecture" dans les manuscrits médiévaux ou : je ponctue, tu lis, il théorise ", Langue française, 40, 32-44. 
MAZZIOTTA, Nicolas (2009) : Ponctuation et syntaxe dans la langue française médiévale. Étude d'un corpus de chartes originales écrites à Liège entre 1236 et 1291 . Tübingen, Niemeyer.

MAZZIOTTA, Nicolas (2018) : "Ponctuation des changements de locuteurs à l'intérieur de l'octosyllabe dans les manuscrits du Chevalier au lion de Chrétien de Troyes». Romania, 136, 300-323.

MORRISON, Stephen (2016) : "Présence et absence de la ponctuation médiévale et moderne dans certains textes anglais de la fin du Moyen Âge : l'embrouillement du sens ", in Valérie Fasseur \& Cécile Rochelois (éds), Ponctuer l'œuvre médiévale. Des signes au sens. Genève, Droz, 489-502.

PARKES, Malcolm B. (1992) : Pause and effect. An introduction to the history of punctuation in the West. Cambridge, Scholar Press.

PIERreVILLE, Corinne [éd] (2016) : Chrétien de Troyes, Le chevalier au lion. Édition bilingue établie, traduite, présentée et annotée par Corinne Pierreville. Paris, Champion.

RoQueS, Mario (1952) : «Le manuscrit fr. 794 de la Bibliothèque Nationale et le scribe Guiot ». Romania, 73, 177-199.

Woledge, Brian (1986) : Commentaire sur Yvain (Le Chevalier au lion) de Chrétien de Troyes. 1. v. 1-3411. Genève, Droz. 